\title{
NANODISPERSE SILICA AND SOME ASPECTS OF NANOTECHNOLOGIES IN THE FIELD OF SILICATE MATERIALS SCIENCE. PART 2
}

\author{
Yu. E. Pivinskii ${ }^{1}$
}

Translated from Novye Ogneupory, No. 12, pp. 28 - 38, December, 2007.

Original article submitted August 14, 2007.

Methodological problems connected with the study of grain size distribution of polyfraction suspensions bearing nanoparticles are considered. Some technological problems of the use of nanoparticles are analyzed. Examples of effective use of nanodisperse kinds of silica for fabricating various silicate materials are presented.

In Part 1 of the paper ${ }^{2}$ we considered the general concepts of nanosystems and generalized recent data on various kinds of silica used in the technology of silicate materials. In Part 2 we will analyze the characteristic features of nanosystems that are determining in the technology of materials that include such systems. An example of silica HCBS will be used for a detailed consideration of general problems connected with the methods of determination of the size distribution of nanoparticles.

\section{COMPLEX ESTIMATION OF DISPERSITY AND GRAIN DISTRIBUTION IN HCBS}

Dispersity and grain distribution of particles of the solid phase play an exceptional role in the technology of materials based on HCBS and especially of quartz ceramics and refractories. This is connected with the fact that in this technology the effect of superclose packing of particles of the solid phase in shaped semiproducts has been implemented for the first time in world practice. Whatever the method of shaping of these materials with the use of cast molding systems (slip casting, electrophoretic or centrifugal shaping, vibration casting of ceramic concretes), it seems possible to obtain a semiproduct with porosity or relative density unattainable in the technologies of other materials. To a great extent this is a result of optimum dispersity and grain distribution of solid-phase particles in the corresponding molding systems. This concerns ceramic materials shaped on the basis of suspensions and ceramic concretes. Molding systems for fabricating ceramic concretes contain a considerable volume

\footnotetext{
1 "NVF KERAMBET-OGNEUPOR" Company, Russia.

2 Published in Novye Ogneupory, No. 11, 2007.
}

fraction of granular or coarse-grained filler (in the form of fused quartz or scrap of quartz ceramics or refractories).

In Fig. 1, the data of [34] are used to plot the grain distribution of HCBS and ceramic concretes characterized by different grain sizes of the fillers. The range of particle sizes in the fillers involves about eight decimal exponents from a fraction of a micron for the colloid component to $10 \mathrm{~mm}$ for the filler. At the same time, the main contribution to the specific surface of both the solid phase of the HCBS (curve $1^{\prime}$ ) and of ceramic concretes (curves $2^{\prime}$ and $3^{\prime}$ ) is made by the very insignificant amount of very fine (highly dispersed) particles (at the level of nanoparticles). The filler ensures only $1-2 \%$ of the specific surface of the solid phase. The corresponding role in the formation of properties of materials based on HCBS (quartz ceramics) and ceramic concretes is played by the reactive colloid component (nanoparticles).

The minimum size $d_{\min }$ of HCBS particles amounts to $\sim 5-10 \mathrm{~nm}$ (nanoparticles), whereas the maximum size $d_{\max }$ attains $100-200 \mu \mathrm{m}$ for HCBS used in the technology of quartz ceramics $[7,35]$ and $300-400 \mu \mathrm{m}$ in the technology of quartz refractories $[27,36]$. In this connection, objective difficulties arise in the study of the fully disperse composition of solid-phase particles in such systems. Therefore, it is expedient to use a complex method consisting of a combination of several individual methods. The study is begun with a wet sieve analysis of a sample of the suspension (commonly from 100 to $200 \mathrm{ml}$ ). In the production of both quartz ceramics and refractories the sieved fraction is estimated in terms of a generalizing characteristic, i.e., the content of particles with size exceeding $63 \mu \mathrm{m}$ (the residue on a sieve with cells $0.063 \mathrm{~mm}$ in size). As applied to suspensions of quartz glass in the production of ceramics this parameter is equal to 

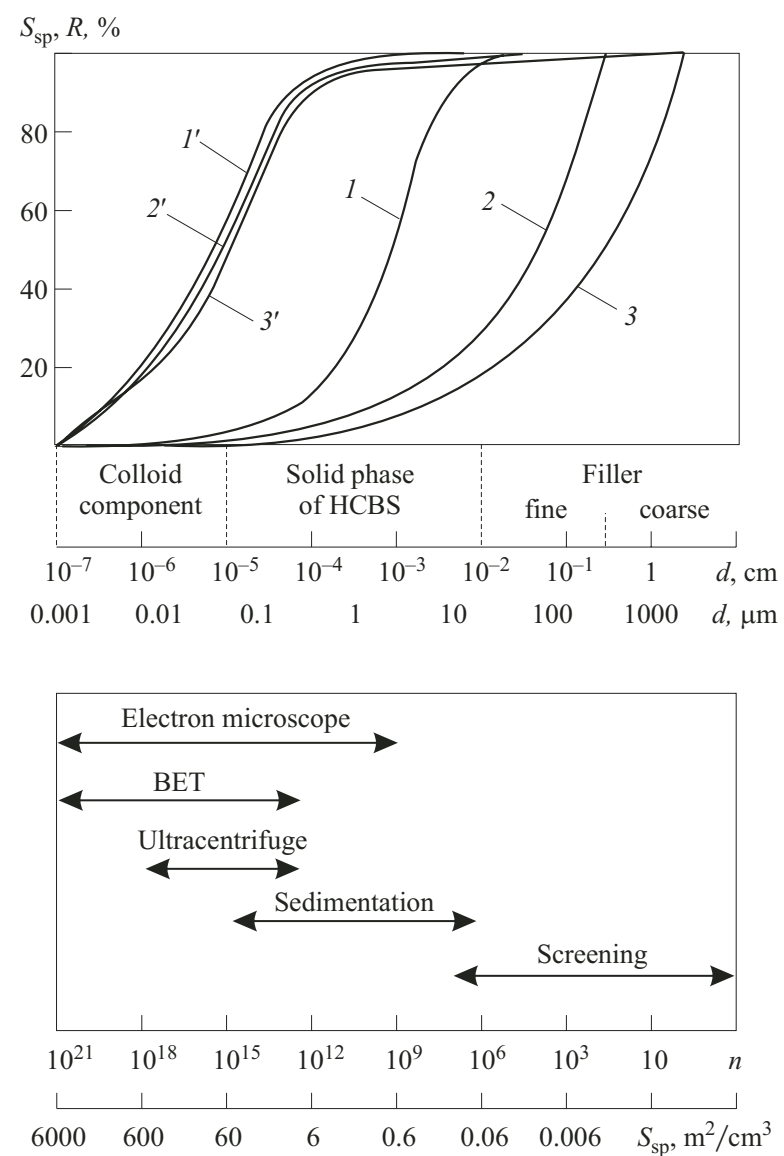

Fig. 1. Integral curves of grain distribution for $\operatorname{HCBS}(1)$, finegrained (2) and coarse-grained (3) ceramic concretes, similar curves of the relative contribution of specific surface $S_{\mathrm{sp}}\left(1^{\prime}, 2^{\prime}, 3^{\prime}\right)$, and general characteristics and experimental methods for determination of various levels of dispersity; $n$ is the number density of the particles (pieces $/ \mathrm{cm}^{2}$ ).
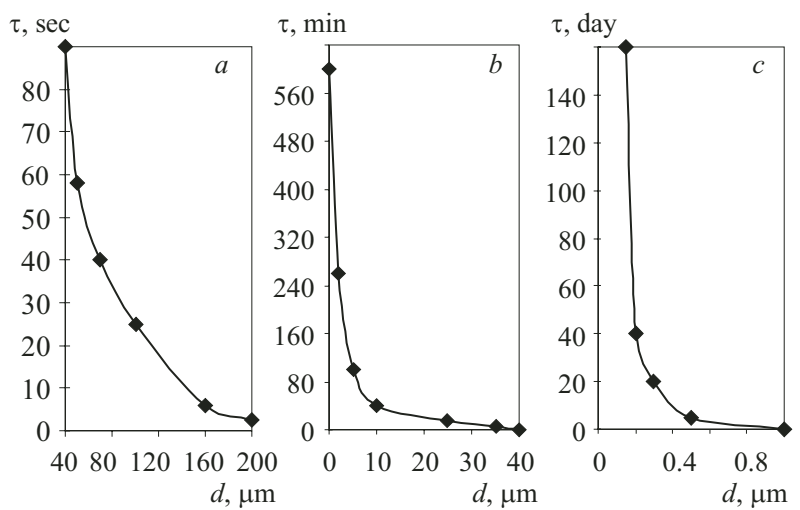

Fig. 2. Dependence of duration $\tau$ of full sedimentation of particles of quartz glass with different diameters $d, \mu \mathrm{m}$, in a column of liquid with a height of $100 \mathrm{~mm}: a, 40-200 ; b, 2-40 ; c, 0.1-1.0$ (the water temperature is $22^{\circ} \mathrm{C}$ ).

$5-8 \%$, and in the production of refractories it is $12-16 \%$. For the purpose of research and for a more detailed study of the processes of fabrication of HCBS it is more expedient to

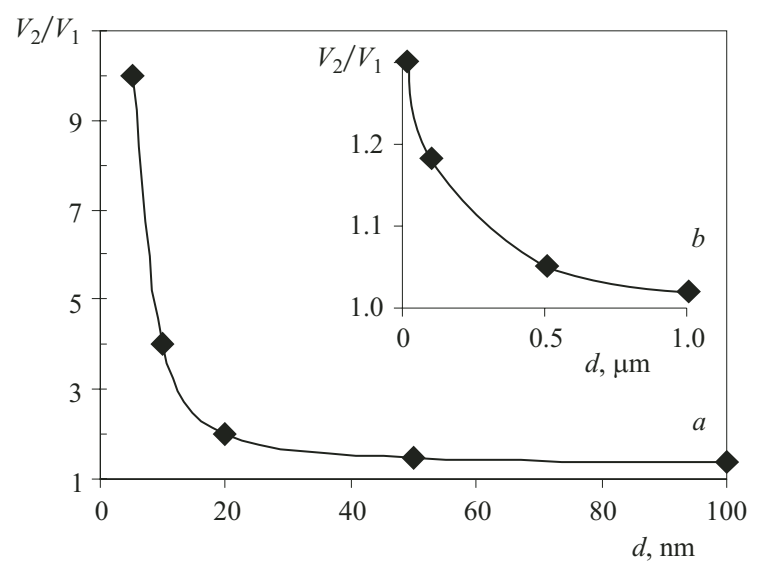

Fig. 3. Dependence of the $V_{2} / V_{1}$ ratio for particles of amorphous silica $5-100 \mathrm{~nm}(a)$ and $0.1-1 \mu \mathrm{m}(b)$ in size.

use a set of sieves that will make it possible to determine the content of narrow groups of factions, i.e., $40-50 \mu \mathrm{m}$ (a sieve with a cell of $40 \mu \mathrm{m}), 50-63(50 \mu \mathrm{m}), 63-100(63 \mu \mathrm{m})$, $100-200 \mu \mathrm{m}, 200-315 \mu \mathrm{m}$, etc. Taking into account the widening of the scale of the "sieve" fractions, it seems possible to perform later a more accurate liquid sedimentation analysis, which is expedient for a suspension sample with $d_{\max }=40 \mu \mathrm{m}$ (i.e., after wet sieving through $40-\mu \mathrm{m}$ cells). This is explainable by the special features of the sedimentation analysis illustrated in Fig. 2.

As a rule, the analysis of deposition of particles in diluted suspensions in performed in a cylinder with liquid column $100 \mathrm{~mm}$ high. It follows from Fig. $2 a$ that the duration of complete deposition of 60-, 100-, and 200- $\mu \mathrm{m}$ fractions is 36,24 , and 3.6 respectively. Therefore, the analysis of sieve fractions $(>40 \mu \mathrm{m})$ by the method of sedimentation leads to a considerable error, because the time of deposition of large particles turns out to be comparable with the initial operations of the analysis (filling of the sample, lowering of the cup, beginning of count, etc.). In order to plot the integral curve after analyzing the suspension, the content of different groups of fractions computed by the conventional method is corrected with allowance for the content of sieve fractions. For example, if the content of sieve fractions is $20 \%$, the actual content of all finer fractions is decreased by $20 \%$ as compared to that computed by the conventional sedimentation methods. In addition, the sedimentation analysis of suspensions with $d_{\max }=100-200 \mu \mathrm{m}$ can also be performed without preliminary screening of the coarse fractions. In this case their content is altered with allowance for parallel screening of the sieves.

Quite considerable difficulties in the analysis of the dispersity of HCBS arise in determination of the size and content of the finest (nanosize) fractions. It follows from Fig. $3 a$ that even for deposition of particles $1 \mu \mathrm{m}$ in diameter the required duration of analysis is $40 \mathrm{~h}(\sim 2$ days $)$. The Tekhnologiya ONPP performs sedimentation analysis 
in the production of quartz ceramics for $24 \mathrm{~h}$. The duration of analysis required for complete deposition of particles with still smaller diameter increases markedly, i.e., to $160 \mathrm{~h}$ for $d=0.5 \mu \mathrm{m}$ and to $4000 \mathrm{~h}$ (42 days) for the upper boundary of the size of nanoparticles $(0.1 \mu \mathrm{m})$. At the same time, it is assumed (and not without reason [9, p. 77]) that as applied to sedimentation analysis the lower boundary of measurable particle sizes is determined by the effect of Brownian motion and is $0.2-0.5 \mu \mathrm{m}$. It should be noted that in the case of the use of a "Sedigraph $5000 \mathrm{D}$ " $\mathrm{x}$-ray sedimentograph the lower bottom of particle sizes is limited to $0.2 \mu \mathrm{m}$. It is known that different variants and methods of sedimentation analysis permit extrapolation and determination of $d_{\min }$ by the method suggested by Tsuryupa [37]. However, the results obtained are quite conventional, while the nanolevel of the fineness of HCBS is a determining one.

For research purposes this problem can be solved by the following method. Conventional sedimentation analysis is performed for 7 days. This time is sufficient for complete sedimentation of particles with diameter exceeding $0.5 \mu \mathrm{m}$. After this a liquid sample in a volume corresponding to its column above the cup is taken from the cylinder with the suspension at a depth of $10 \mathrm{~cm}$ (a variety of pipette method). The mass of the particles suspended in the water after 7 days of settling (the fraction $<0.5 \mu \mathrm{m}$ ) is determined by drying this sample and subsequent weighing of the residual. The entire integral curve of the distribution of particles is corrected with allowance for this mass (as a rule this value is equal to $3-7 \%$ of the mass that settled in 7 days). Since in the time of the sedimentation a substantial part of the particles with a size below $0.5 \mu \mathrm{m}$ settles in a region close to the cup, the total content of this fraction is higher. A more detailed study has shown that the major part of unsettled particles is $0.1-0.5 \mu \mathrm{m}$ in diameter and the rest of the particles have a diameter ranging from $5-10$ to $100 \mathrm{~nm}$. A more exact study of the sizes of the ultrafine particles considered is possible with use of ultracentrifuging, which is described in Part 1 of the present work. Nanoparticles with minimum sizes do not settle even with the help of an ultracentrifuge. Their content is determined by the method of drying of the centrifuged sample of the suspension (liquid).

In some cases (primarily for research purposes) the content and sizes of nanoparticles are determined by the method of long-term (months) settling of the analyzed suspensions subjected to preliminary 10 -fold dilution in cylinders with a height of $200-300 \mathrm{~mm}$. When the settling is finished (fixed time of settling), samples are taken from the cylinder gradually (from top to bottom), and the concentration of particles in them is determined. With allowance for the data on the duration of the sedimentation and on the depth from which the samples were withdrawn we can determine the size of the particles.

However, it should be noted that even this method does not ensure accurate determination of the size and content of nanoparticles. This is explainable by at least two factors.

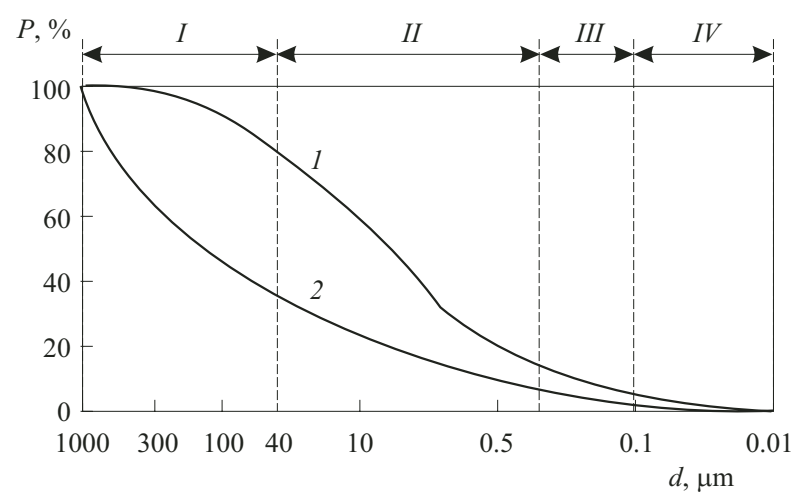

Fig. 4. Integral curves of grain distribution for particles of solid phase in a suspension of fused quartz (1) and in a molding system with granular filler (2); $I-I V$, ranges of grain sizes determined by sieve analysis $(I)$, sedimentation $(I I)$, pipette method (III), and ultracentrifuging $(I V)$.

Based on computations and using the Stokes law we can say that the duration of deposition of particles of amorphous silica (with a density of $2.2 \mathrm{~g} / \mathrm{cm}^{3}$ ) to a depth of $1 \mathrm{~cm}$ is 67 days for particles $50 \mathrm{~nm}$ in diameter and over a year for particles $20 \mathrm{~nm}$ in diameter. At the same time we should remember that at the level of nanoparticles of this size the Brownian motion and the hydration become substantial factors.

It is known that all particles of the solid phase of HCBS are stabilized due to the presence of double or adsorptionsolvate layers that increase the "apparent" or effective diameter of the particles. For medium-size and large particles this effect is insignificant, but for nanoparticles it is quite noticeable (Fig. 3). In accordance with our data the thickness of hydrate layers for silica sols does not exceed $3 \mathrm{~nm}$. Using this value we present in Fig. 3 a dependence of the ratio of the effective volume of particles $V_{2}$ (with allowance for the volume of the hydrate envelope) to the true (not hydrated) volume of sol particles $V_{1}$. It follows from Fig. $3 a$ that as the diameter of the $\mathrm{SiO}_{2}$ particles decreases, the value of $V_{2} / V_{1}$ inceases abruptly. For example, the effective volume of particles $100 \mathrm{~nm}$ in diameter $(0.1 \mu \mathrm{m})$ exceeds their true volume by $19 \%\left(V_{2} / V_{1}=1.19\right)$, whereas for particles $5 \mathrm{~nm}$ in diameter the effective volume grows by more than a factor of 10 . For particles $1 \mu \mathrm{m}$ in diameter the volume of the hydrated layer is equal to about $2 \%$ (see Fig. $3 b$ ), whereas for particles of medium and large sizes its proportion is negligible.

Hydration changes the effective or apparent density of nanoparticles substantially. Even with allowance for the elevated density of bound water (we took the value of $1.20 \mathrm{~g} / \mathrm{cm}^{3}$ for the latter) computed values of $\rho$ amounted to $2.16 \mathrm{~g} / \mathrm{cm}^{3}$ for particles with $d=0.5 \mu \mathrm{m}, 2.04 \mathrm{~g} / \mathrm{cm}^{3}$ for $d=100 \mathrm{~nm}, 1.91 \mathrm{~g} / \mathrm{cm}^{3}$ for $d=50 \mathrm{~nm}$, and $1.44 \mathrm{~g} / \mathrm{cm}^{3}$ for $d=10 \mathrm{~nm}$. With decrease in the apparent density of the particles the difference in the parameters for a hydrated particle and water (one of the factors determining the rate of sedimentation according to Stokes) decreases. For example, for a particle with $d=10 \mathrm{~nm}$ this difference decreases by almost a 
factor of 3 (from 1.2 to $0.44 \mathrm{~g} / \mathrm{cm}^{3}$ ). Due to the special features considered the physical rate of sedimentation of nanoparticles turns out to be considerably or even much lower than follows form the classical Stokes law. In order to illustrate the mentioned features of analysis of particle sizes of HCBS we characterize in Fig. 4 different ranges of particle size from the standpoint of the use of an optimum method of their determination.

\section{DISPERSITY AND DEGREE OF HYDRATION OF NANOPARTICLES: DETERMINING PROCESS FACTOR}

In accordance with $[9,20]$ particles of silica hydrosol have a structure similar to that of globules of water-soluble polymers. Due to the lyophilic behavior the boundary between the internal surface layer of a particle and its external part is smeared. It follows from Fig. 5 that the micelle includes a nucleus, a layer of potential-determining $\left[\mathrm{SiO}_{3}\right]^{2-}$ ions, and an adjoining layer of most strongly held counterions of $\mathrm{H}^{+}(n-x)$, near which lies a diffusion layer that contains the rest of the $\mathrm{H}^{+}$counterions. Thus, the micelle consists of a nucleus and an ionogenic part formed by two ion layers, i.e., adsorption and diffusion ones.

It is obvious from this scheme that silica hydrosols are highly lyophilic dispersions. Their aggregative stability in the acid range is determined primarily by the hydrophilic behavior of the $\mathrm{SiO}_{2}$ surface capable of forming hydrogen bonds that are responsible for formation of boundary polymolecular hydrated layers. In accordance with $[9$, p. 253] the thickness of the hydrate layer $\delta$, which consists of $\mathrm{H}_{2} \mathrm{O}$ molecules bonded through hydrogen bonds to the surface of the particles, amounts to $3-10 \mathrm{~nm}$. The presence of this layer gives rise to forces of interaction between the particles under the condition of overlapping of the surface layers. These forces, which are known as structural ones, ensure the existence of a structural component of wedging pressure.

From the standpoint of economical expedience producers and consumers of silica sols strive to produce and to use highly concentrated systems that are stable for at least a year. We use the data of [19] to show in Fig. 6 the dependence of the maximum possible concentration of stabilized aqueous silica sols on the diameter of the particles. The parameters of the maximum possible concentration are affected considerably by the diameter of the particles. For example, when this diameter increases from 10 to $50 \mathrm{~nm}$, the volume fraction (see curve $C$ in Fig. 6) grows from 12 to $45 \%$. This is explainable as follows. If we assume that the thickness of the hydrate layer $\delta$ for particles of any size is constant and equal to $2.5 \mathrm{~nm}$, then, with allowance for this value, the initial diameter $d=10 \mathrm{~nm}$ increases to $15 \mathrm{~nm}$ and the initial diameter $d=50 \mathrm{~nm}$ increases to $55 \mathrm{~nm}$. However, the effective volume of the sol (with allowance for the bound water) in the former case increases by a factor of 3.7, while in the latter case it increases only by a factor of 1.33 . For this example,

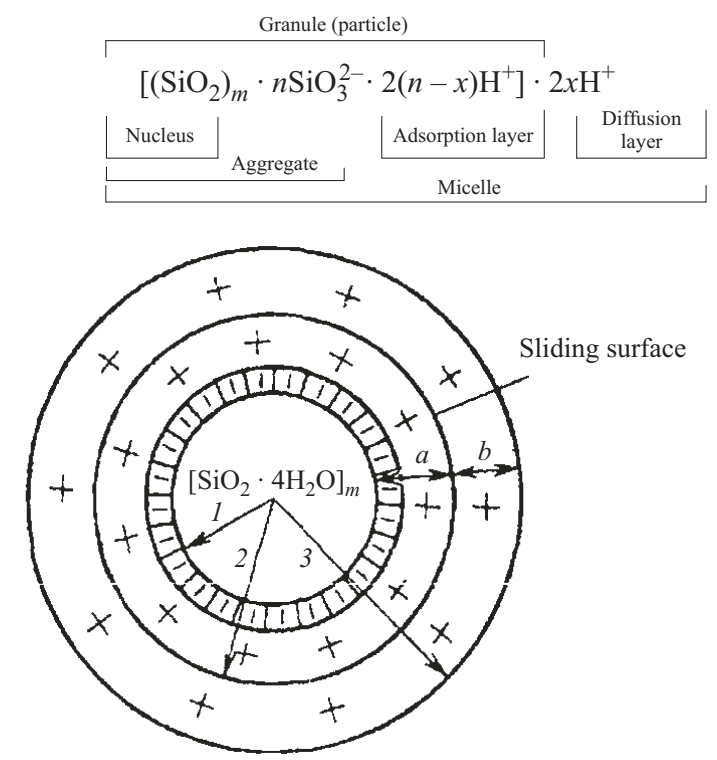

Fig. 5. Scheme of the structure of silica sol micelle: $(-)$, potentialdetermining ions of $\mathrm{SiO}_{3}^{2-} ;(+)$, counterions of $\mathrm{H}^{+} ; m$, number of $\mathrm{SiO}_{2} \cdot y \mathrm{H}_{2} \mathrm{O}$ molecule in the nucleus; $a$, adsorption layer; $b$, diffusion layer; 1 , nucleus; 2 , colloid particle; 3 , micelle.

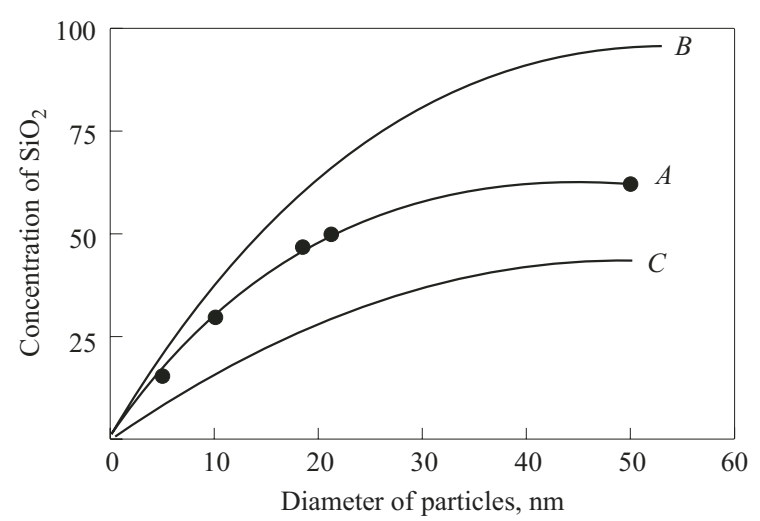

Fig. 6. Maximum concentration of stabilized aqueous silica sols as a function of particle size at $\mathrm{pH} \sim 9.5$ : $A$, concentration in mass percent; $B$, concentration in grams of $\mathrm{SiO}_{2}$ per $100 \mathrm{ml}$; $C$, volume faction of $\mathrm{SiO}_{2}(\times 100)$.

with allowance for the effective volume of the particles, the maximum concentration of silica sol for particles with an initial diameter of $10 \mathrm{~nm}$ grows from 12 to $44 \%$ and that of particles with an initial diameter of $50 \mathrm{~nm}$ grows to $62 \%$. In all probability, the values of $\delta=3-10 \mathrm{~nm}$ for silica sols presented in [9] are overestimated. If we assume that for a silica sol with initial $d=10 \mathrm{~nm} \delta=5 \mathrm{~nm}$ (i.e., the diameter in the hydrated condition is $20 \mathrm{~nm}$ ), then at $C_{V}=12 \%$ (see curve $C$ in Fig. 6) the effective volume would have grown by a factor of 8 and the effective concentration would have attained $100 \%$. The value of the effective volume concentration (with allowance for $\delta$ ) for monodisperse suspensions of silica sols cannot exceed 55\%. Taking these results into account we can 
expect that the actual values of $\delta$ can hardly exceed $3 \mathrm{~nm}$, which corresponds to $8-12$ monolayers of water.

One of the problems of the use of silica sols is preservation of integrity and continuity of the film during drying (transformation of the sol into a dried gel). Cracks may form in the dried gel due to considerable shrinkage stresses. In this case it is expedient to use polydisperse silica sols. According to Iler [19, p. 505], when the composition of a silica sol is polydisperse (particles 100, 22, and 7-10 nm in size), drying defects are absent. The gaps between coarse particles in a dried gel are filled by smaller particles and this lowers the stresses arising due to the drying quite substantially (Fig. 7).

The examples considered lead us to understanding the problem of practical use of nanoparticles. The problem is that as the dispersity of the nanoparticles increases, so does the degree of their hydration, which is accompanied by decrease in the maximum possible concentration of the molding systems based on them.

At the same time, all categories of bound water in the process of fabrication of materials should inevitably be removed in different stages of the process. Independently of the composition of particles of the solid phase of the suspension all of them are stabilized due to the presence of binary ion or adsorption-solvate layers that increase the apparent or effective diameter of the particles. It is known [36, p. 112] that in accordance with the energy criterion bound water is held in the system due to chemical and physicochemical forces $(800-0.1 \mathrm{~kJ} /$ mole) that act from the side of surface particles. These forces change the mobility and the properties of the water as compared to the free water in the volume. From the standpoint of the production process it is convenient to subdivide the whole of the bound water in the hydrated structure of nanoparticles into physically bound and chemically bound (constitutional) varieties (Fig. 8). The former is removed by drying $\left(100-110^{\circ} \mathrm{C}\right)$, whereas dehydration of the chemically bound water occurs until high temperatures $\left(1000-110^{\circ} \mathrm{C}\right)$.

Removal of bound water is accompanied either by considerable shrinkage in drying (physically bound water) or by substantial growth (in comparison with the dried semiproduct) in the additional porosity $P_{\mathrm{o} \text { ad }}[13,15]$ due to dehydration of the material at high temperatures (chemically bound water). It has been noted in [7] that even relatively low (up to $0.2-0.5 \%$ ) shrinkage of preforms from quartz ceramics in drying due to the elevated dispersity of suspensions makes it impossible to obtain quality large-size castings for rocket cowls by the method of slip casting. However, as applied to the process of drying of semiproducts based on highly dispersed materials the main problem is not only the result of shrinkage. It is known that the removal of free water from a gel gives rise to wetting capillary meniscuses that cause the appearance of additional (Laplace) pressure [9]. The latter depends on the curvature of surface in the pores, which is determined in its turn by the size of the nanoparticles. Therefore, in the stage of drying the capillary forces cause cracking of the spatial structure of the gel [9, p. 278].

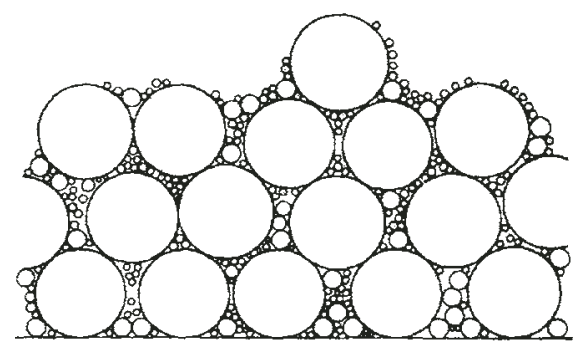

Fig. 7. Scheme of formation of a strong and dense gel from a mixture of large and small silica sol particles.

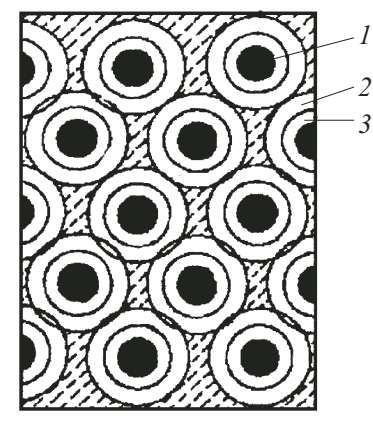

Fig. 8. Structure of hydrated nanoparticles: 1 , nanoparticle; 2 , physically bound water; 3 , chemically bound water.

In order to lower the effect of the capillary pressure it is recommended to perform the drying under vacuum or to treat the gel preliminarily by a chemical reagent [9].

As an illustration of the topics considered we will discuss the data of the authors of [27, p. 133] on fabrication and properties of suspensions based on nanodisperse silica in the form of aerosil with $S_{\mathrm{sp}}=300 \mathrm{~m}^{2} / \mathrm{g}\left(d_{\mathrm{av}}=10 \mathrm{~nm}\right)$. This silica was used for preparing aqueous suspensions with maximum volume faction $C_{V}=0.14$ characterized by considerable dilation properties. Castings on the basis of these suspensions shrink considerably (by 12\%) when dried. The porosity of the specimens after drying was $60 \%$.

It should be noted that suspensions of colloidal nanoparticles separated from quartz glass HCBS possess an equally low concentration and high viscosity. It is noted in [7] that after 7 years of settling of quarts glass suspensions a silica gel formed on the interface of the sediment and the silica sol. At $C_{V}=0.13$ it was characterized by considerable yield strength $(2 \mathrm{~Pa})$ and well manifested thixotropy of flow (upon growth in the stress from 2 to $40 \mathrm{~Pa}$ the viscosity decreased by a factor of 30 ).

Similar dependences are typical for suspensions based on bentonite clays that can be treated quite legitimately as nanodisperse ones (the value of $S_{\mathrm{sp}}$ attains several hundred square meters per gram). A study of such a clay (a montmorillonite) [27, p. 312] has shown that suspensions based on it possess a substantial yield strength even at $C_{V}$ equal to 0.09 and 0.11. At the maximum value $C_{V}=0.19$ the yield strength is $50 \mathrm{~Pa}$ (a solid-like structure). Castings based on these suspensions have linear shrinkage in drying exceeding $20 \%$. 
A study of a colloid component separated by stripping of centrifugate from a quartz sand HCBS has shown that its $S_{\mathrm{sp}}=260 \mathrm{~m}^{2} / \mathrm{g}$, and the loss in the mass due to calcination attains $12 \%$. Computations made with the use of these data have led to the following important conclusion: in any case $1 \mathrm{~m}^{2}$ of a surface binds about $0.45 \mathrm{mg}$ liquid, which corresponds to the thickness of a layer of about $0.5 \mathrm{~nm}$ (two monomolecular layers) of chemically bound liquid [12]. The thickness of the layer of physically bound liquid removed in drying is presumably $1-2 \mathrm{~nm}$.

As applied to the ceramics technology with the use of dry nanoparticles or nanosystems we should take into account the following specific factors. As the size of the particles decreases, the packing density of the powders (particles) decreases abruptly due to the fact that the cohesion forces that arise upon decrease in the interparticle distance grow abruptly relative to their mass [14]. The density of semiproducts obtained by different methods also depends considerably on these factors. Therefore, fabrication of high-density ceramics from nanopowders is accompanied by high volume shrinkage in sintering (up to $50-60 \%$ ), which is quite unfavorable for the production process [14].

This allows us to infer that fabrication of the materials in question with predominant use of nanoparticles is very difficult technologically and inexpedient economically in the majority of cases. A real and very effective direction in a wide domain of the science of silicate materials consists in developing processes based on the use of an optimum content of nanoparticles. Specifically, this has been demonstrated for numerous materials based on HCBS [7, 12 - 15]. The initial molding systems for such materials contain a small (optimum) amount of nanoparticles and are characterized by considerable polydispersity because they are represented by both relatively coarse particles obtained by dispersion $(0.05-0.3 \mu \mathrm{m})$ and by fine particles $(0.01-0.02 \mu \mathrm{m})$ formed in the process of polycondensation of silicic acid. This ensures constancy of the volume of the materials in the stage of their drying and heat treatment (in roasting or in service). In the technologies of HCBS and ceramic concretes nanoparticles produce a complex positive effect. In the stage of fabricating HCBS and molding systems they promote attainment of optimum flow characteristics and in the stage of roasting or service the optimum content of nanoparticles determines substantial growth in the strength even at $800-1000^{\circ} \mathrm{C}$ and makes it possible to form a thin-pore microstructure, which is usually impermeable for melts [14, 27].

Thus, the nanosize features of a structure are not always determining ones when we deal with creation of silicate materials. For most materials, especially for bulk (massive) ones, it is expedient to consider different levels of structure from nanometer ones to millimeter ones and to understand that they are closely interrelated. In some cases the determining role belongs to fragments with micrometer and even millimeter sizes (for example, for refractory and building concretes) rather than to nanometer fragments. Therefore, when creating new materials we should strive to ensure optimum sizes of the fragments of their structure [4, 27].

\section{NANOSILICA IN THE TECHNOLOGY OF SILICATE MATERIALS}

Nanodisperse silica in various forms is used already quite widely in the production of some ceramic, refractory, and building materials. It is possible that the most vivid examples of its efficiency are data on the effect of the content of nanoparticles in HCBS with silica composition on their binding properties.

The effect of the concentration of colloid component (nanoparticles) $C_{\mathrm{c} . \mathrm{c}}^{\mathrm{red}}$ on the strength and density of the material is presented in [12] for HCBS of quartz sand. In order to vary $C_{c . c}^{\text {red }}$ the systems studied were enriched with or depleted of the colloid component so that the value of $C_{\text {c.c }}^{\text {red }}$ ranged within $0.01-2.22 \%$. According to the data of the method of low-temperature adsorption of nitrogen the colloid component was characterized by $S_{\mathrm{sp}}=260 \mathrm{~m}^{2} / \mathrm{g}\left(d_{\mathrm{av}}^{\mathrm{p}}=9.5 \mathrm{~nm}\right)$ and the volume fraction of the constitution water $W_{x}=16 \%$.

The results of the study of the effect of the content of colloid component on the properties of castings are generalized in Fig. 9. The figure reflects its exceptionally strong effect primarily of the strength properties that characterize the binding capacity of the HCBS. It can be seen that $\sigma_{\text {bend }}$ between the extreme values of $C_{\text {c.c }}$ differs by about a factor of 60 . The porosity of the casting also changes considerably. In the given case we observe that the value of $P_{\mathrm{o}}$ cast is the lower the higher the value of $C_{\text {c.c }}$. For an ultimately enriched suspension $P_{\mathrm{o} \text { cast }}=11 \%$, whereas for the ultimately depleted suspension it increases to $18 \%$. The effect of $C_{\text {c.c }}$ in this case seems to be explainable by the "lubricating" action of the colloid component.

Analyzing Fig. 9 we will see that the strength of the castings is not proportional to $C_{\mathrm{c} . \mathrm{c}}$. Even when the value of $C_{\mathrm{c} . \mathrm{c}}$ grows by a factor of 3 (from 53 to $160 \mathrm{~g} / \mathrm{l}$ ) the value of $\sigma_{\text {bend }}$ increases insignificantly (from 4.6 to 6.2 MPa). Decrease in $C_{\text {c.c }}$ against the initial value causes substantial lowering of the strength (by a factor of $3-5$ ), which cannot be associated only with the growth in the porosity of the castings. This reflects the dominant effect of the content of colloid component in a suspension on its binding properties.

Undoubtedly, the mechanism of solidification of HCBS is also determined to a considerable degree by the same factor. A certain (yet undetermined) fraction of the colloid component of HCBS consists of silicic acids and sols formed in the system upon polymerization. It is necessary to determine the proportion of sols formed due to dispersion and due to condensation in the colloid component as well as their contribution into the binding properties.

We presume that the mechanism of solidification and strengthening of materials based on HCBS of acid or acid-amphoteric compositions is a result of formation 
of intergrain contacts based on hydrogen bonds of the locking $\mathrm{O} \cdots \mathrm{H}-\mathrm{O}-\mathrm{H} \cdots \quad$ water molecules,

$$
\equiv \mathrm{Si}^{\mathrm{OH}} \leftarrow: \underbrace{-\mathrm{H}}_{\mathrm{H}}=
$$

donor-acceptor interaction, and

$\equiv \mathrm{Si}-\mathrm{O}-\mathrm{Si} \equiv$ contacts of a polycondensation nature $[13,36]$. Accordingly, an important role in the solidification mechanism belongs to the existence of reaction-capable silanol groups on the surface of particles of the solid phase of HCBS.

The silanol groups transform into siloxane groups in the process of polycondensation drying due to heating, when two silanol groups give rise to one siloxane group and a water molecule in accordance with the equation

$$
\equiv \mathrm{Si}-\mathrm{OH}+\mathrm{HO}-\mathrm{Si} \equiv \stackrel{t}{\longrightarrow} \equiv \mathrm{Si}-\mathrm{O}-\mathrm{Si} \equiv+\mathrm{H}_{2} \mathrm{O} .
$$

This is confirmed by the substantial strengthening of castings dried at $100-140^{\circ} \mathrm{C}$. As applied to the understanding of the mechanism of the binding properties of HCBS, data on the kinetics of condensation and gel formation in solutions of silicic acids during synthesis of silica sols can be important [20].

The content of nanoparticles in HCBS strongly affects the pore structure of materials based on them. It follows from the data of Fig. 10 that the pore structure can be changed considerably by changing the content of nanoparticles in the initial HCBS. At maximum $C_{\text {c.c }}$ (see curve 1 in Fig. 10) the structure has primarily nanosize pores less than $60 \mathrm{~nm}$ in diameter. Even the maximum pore diameter does not exceed $100 \mathrm{~nm}$.

Let us consider the use of fine amorphous silica known as microsilica (MS) as an example of efficient use of nanodisperse $\mathrm{SiO}_{2}[15,18]$. This kind of silica is introduced as an additive into constructional or low-cement refractory concretes (LCRC) [15]. An important factor from the standpoint of economy and ecology is the fact that MS is a waste of the production of silica, ferrosilicon, and silicomanganese [18]. Today the use of the ultrafine waste of the production of ferroalloys and crystalline silicon as active microfiller for structural concretes solves many important problems of the building industry, i.e., fabrication of high-strength, superdense, and long-life concrete and reinforced concrete structures, reduction of energy consumption in the production of concrete, and, if necessary, saving of cement [18].

In accordance with [38] the efficiency of silica additives in the nanotechnology of cement composites is explainable by the fact that the nanoparticles of $\mathrm{SiO}_{2}$ react with calcium hydrate and form calcium hydrosilicate. This causes a substantial decrease (by up to 30\%) in the content of pores with a size exceeding $1 \mathrm{~nm}$. For structural concretes the MS should contain $80-90 \% \mathrm{SiO}_{2}$; in the LCRC technology its purer varieties are used. In world practice the most widely used MS is

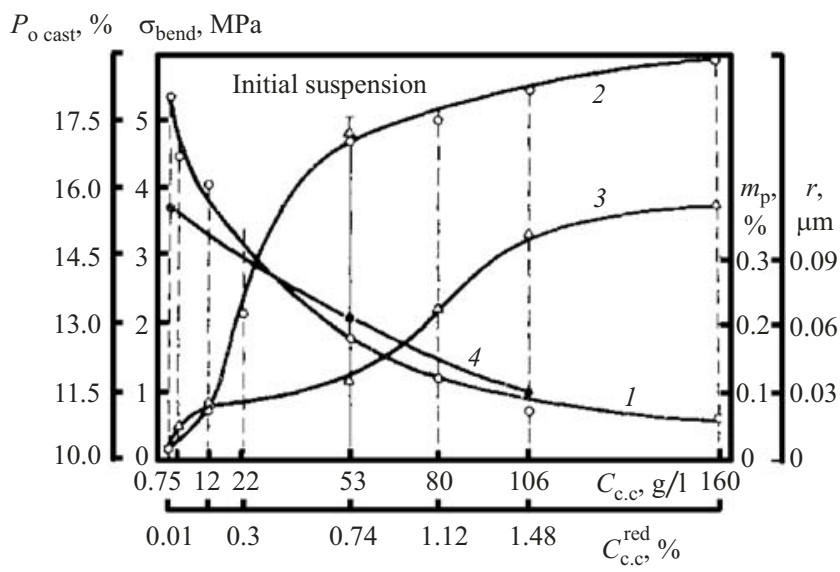

Fig. 9. Effect of $C_{\mathrm{c} . \mathrm{c}}$ and $C_{\mathrm{c} . \mathrm{c}}^{\text {red }}$ in the initial quartz sand HCBS $\left(C_{V}=0.73\right)$ on $P_{\mathrm{o} \text { cast }}(1)$ and $\sigma_{\text {bend }}(2)$, loss in the mass $m_{\mathrm{p}}(3)$, and mean radius of the pores $r(4)$ of castings based on them.

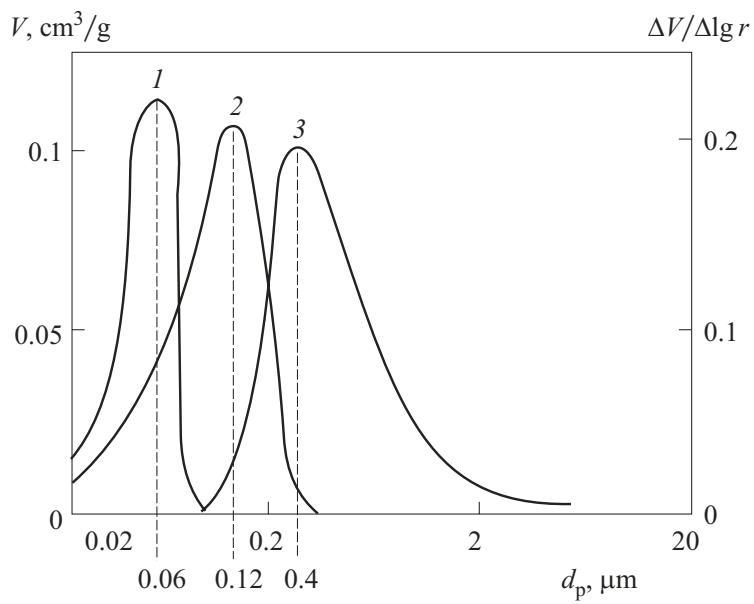

Fig. 10. Differential programs of a binder (casting) based on quartz glass HCBS with the content of colloid $(\leq 200 \mathrm{~nm})$ component $C_{\mathrm{c} . \mathrm{c}}^{\text {red }}$ equal to 7.5 (1), 3 (2), and 0.7 (3) percent.

produced by "Elkem Microsilica" in Norway. The content of $\mathrm{SiO}_{2}$ in this MS is $96.0-97.5 \%$ and $S_{\mathrm{sp}}=20 \mathrm{~m}^{2} / \mathrm{g}[15,27]$.

As applied to the technology of LCRC an additive of microsilica produces a multifunctional effect that consists in improving both the flow characteristics of the initial molding systems and the properties of the refractory concretes obtained with this additive. It is known that the size of the finest particles in the matrix systems of LCRC (reactive alumina, HAC) is equal to $0.5-0.1 \mu \mathrm{m}$. It is schematically shown in Fig. 11 that in this case the introduced particles of microsilica are a kind of microfiller. When mixed (suspended), they pack in the gaps between relatively coarse particles of the matrix system and this is accompanied by squeezing of a part of the dispersion medium (see Fig. 11). Thus, by introducing microsilica microfiller we should be able to lower the viscosity of the molding system at the same moisture content or to reduce the moisture content at the same viscosity as in the system without additive. 


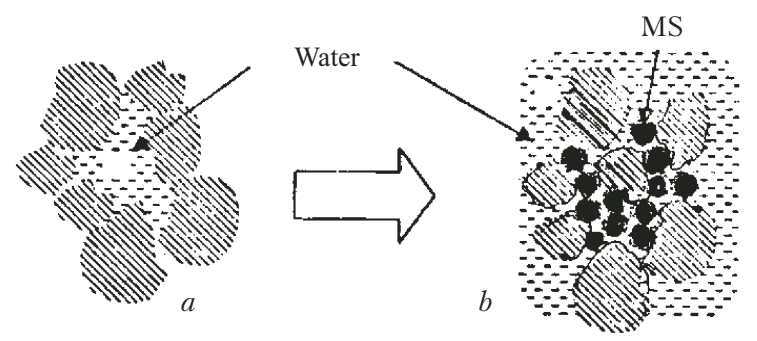

Fig. 11. Effect of MS used as microfiller in concentrated suspensions: $a$, initial suspension; $b$, suspension after the introduction of MS.

A necessary condition of the process in question (see Fig. 11) is the introduction of effective deflocculating agents (liquefiers) and intense mixing. If we take into account the general concepts of rheology and colloid chemistry [36], the efficiency of introduction of MS will be explainable by redistribution of the dispersion medium $C_{W}$ in the molding system. The state describable by the diagram presented in Fig. 1 is characterized by a considerable volume of kinetically free liquid $C_{W s}$, i.e., the mechanically captured fraction of $C_{W s}$ due to relatively loose packing. The introduction of MS lowers the fraction of $C_{W s}$ and is accompanied by proportional growth in the fraction of kinetically free liquid $C_{W k}$. The critical concentration of the system $C_{V c r}$ increases too.

Introduction of 3-5 mass \% microsilica into refractory concretes with high-alumina and corundum compositions promotes relatively low-temperature strengthening due to formation of mullite at the temperatures in question [15, 27]. In the technology of HCBS and ceramic concretes of high-alumina composition the effect of the introduction of MS has been implemented using a principally new and more effective process [39]. At first, an optimum amount (10\%) of highly disperse quarts glass (HDQG) was introduced into HCBS with mullite or high-alumina (bauxite) composition by "pouring" the waste of the production of steel-teeming refractories produced by the method of centrifugal casting [13]. Then the HDQG was "treated" right in wet milling of bauxite HCBS due to the effect of selective disintegration [7, p. 215]. Therefore, the introduced quartz glass $(10 \%)$ concentrated in the final milling stage in the finest (up to $0.5-2.0 \mu \mathrm{m}$ ) fractions. A considerable part of the HDQG was represented by nanoparticles $(<0.1 \mu \mathrm{m})$. Thus, in contrast to MS, which is usually characterized by an elevated content of impurities and low polydispersity, the HDQG obtained by joint milling contained an elevated amount of $\mathrm{SiO}_{2}$ (99\%) and had considerable polydispersity [39].

The advantages of the use of HDQG additives obtained directly in wet milling of HCBS as compared to MS additives are obvious and consist in the following:

- the polydispersity and the presence of a considerable amount of nanoparticles in the HDQG (as compared to MS) intensifies the effect of the microfiller, which results in lower viscosity and dilation at comparable concentrations. It has been shown in $[27,39]$ that $C_{V c r}$ in high-alumina HCBS with optimum content of HDQG can attain $0.84-0.86$ (the poro- sity of castings from mixed HCBS is reduced to $13.5-$ $16.0 \%)$ :

- as a result of formation of structure in the matrix system the HCBS bearing HDQG acquire a finer pore structure, because the latter is largely determined by the presence of nanoparticles in the system;

- the elevated degree of dispersion of the HDQG (as compared to MS) ensures a lower temperature of the beginning of mullite formation in high-alumina systems and the strengthening of the material occurs at a lower temperature too;

- HDQG include a minimum content (up to $0.20-$ $0.36 \%)$ of undesirable admixtures $\left(\mathrm{Fe}_{2} \mathrm{O}_{3}, \mathrm{Na}_{2} \mathrm{O}\right.$, etc. $)$, the content of which in MS amounts to $0.6-1.0 \%$. For this reason the concretes obtained with the use of HDQG should possess improved thermomechanical properties. This assumption is based on the known excremental data on the effect of the purity of MS on the high-temperature strength of SLCRC [27].

In wet milling of HCBS the positive effect of ultrafine particles (the colloid component) is a result of the fact that their presence ensures a high rate of milling due to the "lubricating" action [38]. As applied to bauxite HCBS bearing quarts glass the effect caused by nanoparticles in the stage of wet milling and stabilization (dilution) manifests itself in their heterostabilizing action with respect to the main part of the particles. A detailed analysis of this effect is made in [14].

As applied to high-alumina $\mathrm{HCBS}$ bearing $\mathrm{SiO}_{2}$ nanoparticles an important role is played by the processes of acid-base interaction [38]. It is known that the reactions of acid-base interaction between solid and liquid phases occur on their interface; therefore, as has been shown in [27, 38], the process is controlled not only by the volume proportion of the components but also by the size of the interface. Even a relatively low content of $\mathrm{SiO}_{2}$ nanoparticles in these systems transfers them, in accordance with the classification given in [13], from an acid-amphoteric variant of HCBS to an acid variant, which is accompanied by considerable worsening of the flow properties.

Cement-free refractory concretes obtained on the basis of a colloid-gel suspension [29] contain about $10 \%$ silica sol binder; with allowance for its $40 \%$ concentration this amounts to about $4 \%$ dry substance in the mass of predominantly high-alumina concrete. In all probability, the silica sol binder in such mixes also plays the role of a stabilizer. Mullite forms in service of concretes with such composition. As compared to similar low-cement concretes, these concretes have process advantages (the possibility of pumping and self-spreading at low moisture content) and improved thermomechanical properties in service.

The use of insignificant $(0.6 \%)$ additives of nanodisperse silica (aerosil) in a quartz glass suspension improves its resistance to sedimentation markedly [35, p. 113]. It has been assumed that with allowance for the $200-300$-fold difference in the dispersity of aerosil and of solid-phase particles of the suspension due to the introduction of the additive, the 
total dispersity of the suspension increases. This hinders the displacement of relatively large quartz glass particles and, possibly, leads to the appearance of ultimately low shear stresses that are sufficient for stability of the suspension. An example of an exceptionally high effect of even a low $(0.75 \%)$ additive of "Hardness-M" nanodisperse silica sol composition on the properties of heavy fine-grained concrete is given in [38]. The introduction of this plasticizing additive has reduced the porosity of the concrete by $40 \%$. The ultimate bending strength was doubled and the frost resistance was more than doubled. For the case of cement consumption of $950 \mathrm{~kg} / \mathrm{m}^{3}$ the ultimate compressive strength attained $106 \mathrm{MPa}$. The shrinkage of concretes with this additive also decreases many times $(0.3 \mathrm{~mm} / \mathrm{m}$ against $3.7 \mathrm{~mm} / \mathrm{m})$.

Considerable experience in the use of sol-gel compositions based on ethyl silicates is generalized in [30] for the technology of ceramic and refractory materials. The main process stages in the sol-gel methods of fabrication of materials based on silica sols are described in $[9,20]$.

A recent example of high efficiency of the use of nanodisperse silica has been reported at UNITECR 07 in September 2007 [40]. The "Degussa" Company (Germany) has developed liquid organomodified siloxanes that acquire a structure with chemical linking after drying. The process is accompanied by a $45 \%$ loss in the mass at $100-650^{\circ} \mathrm{C}$. "Degussa" produces several kinds of reactive organosiloxanes traded as "Dynasylan HYDROSIL" and used for surface treatment of minerals, reinforcing fiber glass, and as an additive for water-soluble polymer systems. It is quite possible that the authors of [40] have used additives of this class.

As applied to fabrication of corundum refractories the introduction of a highly reactive siloxane binder ensures con- siderable hardening of the material. For example, after a heat treatment at $600-800^{\circ} \mathrm{C}$ the ultimate compressive strength of the refractories attains $70 \mathrm{MPa}$. In the range of 1000 $1300^{\circ} \mathrm{C}$ the amorphous silica transforms into cristobalite, after which mullite forms.

The examples described prove that the use of various kinds of nanodisperse silica in the production of ceramic, refractory, and building materials is very effective. From the standpoint of engineering and economy the use of nanodisperse materials seems expedient in the form of insignificant additives or as one of the components $(5-10 \%)$ of molding systems.

\section{(To be continued)}

\section{REFERENCES}

34. Yu. E. Pivinskii, "A study of vibration molding of ceramic concretes. Molding systems and main regular features of the process," Ogneupory, No. 6, 8-14 (1993).

35. Yu. E. Pivinskii and A. G. Romashin, Quartz Ceramics [in Russian], Metallurgiya, Moscow (1974).

36. Yu. E. Pivinskii, Theoretical Aspects of the Technology of Ceramics and Refractories, Selected Works, Vol. 1 [in Russian], Stroyizdat, Moscow (2003).

37. N. N. Tsuryupa, A Practicum on Colloid Chemistry [in Russian], Vysshaya Shkola, Moscow (1963).

38. P. G. Komokhov, "Sol-gel as a concept of the nanotechnology of cement composite," Stroit. Mater, No. 8, 14 - 15 (2006).

39. Yu. E. Pivinskii and A. D. Dobrodon, "Fabrication and properties of binders for high-alumna suspensions in the "bauxite quartz glass' system,” Novye Ogneupory, No. 5, 19 - 26 (2002).

40. Th. Ebbrecht, B. Weyershausen, T. R. Lipinski, et al., "A novel high performance binder for refractories," Proc. of UNITECR 07, Dresden, 2007, pp. $388-390$. 